\title{
Sondage du Grand Moncton
}

Dans le cadre de la deuxième stratégie d'immigration, les leaders de Moncton se sont adressés aux membres du public pour prendre la mesure de leur compréhension en ce qui concerne l'immigration et pour solliciter leurs commentaires sur la planification de l'avenir de la ville. Vous trouverez ci-dessous le sondage d'opinion publique qu'on a diffusé lors de la phase de consultation de la stratégie d'immigration. Ce sondage d'opinion illustre bien les mythes au sujet d'immigration qu'il faut réfuter ainsi que les faits qu'il faut accentuer. En utilisant la feuille de travail " Être à la mesure " ainsi que votre modification de ce sondage, vous serez capable également de prendre le pouls de la ville en matière d'immigration.

1] Vrai ou faux : Le nombre de gens nés au Canada et travaillant au Nouveau-Brunswick a été en augmentation depuis 2008.

$>$ Vrai

$>$ Faux

$>$ Indécis

2] Entre 2011 et 2016, les immigrants représentaient quel pourcentage de la croissance démographique du Grand Moncton?

$>62 \%$

$>51 \%$

$>12 \%$

$>43 \%$

3] Vrai ou faux: Plus de 100 des entreprises du Grand Moncton appartiennent à des immigrants.

$>$ Vrai

$>$ Faux

$>$ Indécis

4] Vrai ou faux : Le taux de chômage de la main-d'œuvre immigrante est plus élevé que celui de l'ensemble de la population.

$>$ Vrai

$>$ Faux

$>$ Indécis

5] Combien y a-t-il d'étudiants internationaux inscrits dans les établissements post-secondaires de notre région?

> Plus de 1000

$>$ Moins de 1000

$>$ Indécis

6] Ces dernières années, le taux d'immigration du Grand Moncton a plus que :

> doublé

$>$ triplé

$>$ Indécis
7] Dans les comtés de Westmorland et d'Albert, combien y a-t-il de travailleurs âgés de plus de $\mathbf{5 5}$ ans?

$>5000$

$>10000$

$>20000$

8] Dans le Grand Moncton, quels sont les secteurs menacés par la diminution de la main-d'œuvre ?

$>$ Finance et assurances

$>$ Fabrication

> Transport et entreposage

> Technologie informatique

> Soins de santé

$>$ Tourisme

9] Dans le Grand Moncton, combien de postes ont été comblés grâce à des foires aux emplois visant des étudiants internationaux et des nouveaux arrivants ?

$>$ Moins de 100

$>123$

$>210$

> Plus de 250

10] Vrai ou faux : Dans le Grand Moncton, le taux de travail autonome est plus élevé parmi les immigrants.

$>$ Vrai

$>$ Faux

$>$ Indécis

11] En 2018, le nombre d'étudiants internationaux inscrits dans l'Université de Moncton et l'Université Mount Allison représentait quel pourcentage du nombre total d'étudiants universitaires internationaux dans la province?

$>38 \%$

$>12 \%$

$>44 \%$

12] Combien y a-t-il d'associations ethnoculturelles dans le Grand Moncton?

$>$ Moins de 10

$>15$

$>$ Plus de 20 